\title{
ALICANTE, ENCRUCIJADA DE CAMINOS
}

Alfredo Morales Gil

\section{RESUMEN}

La posición geográfica que ocupa la ciudad de Alicante en el litoral del sureste peninsular viene justificada por la convergencia sobre este lugar de dos vías naturales de penetración hacia el interior y su contacto con el Mediterráneo, al abrigo de los relieves bélicos que la libran de las incidencias puntuales de los vientos de levante, de manera que la bahía junto a la que se asienta permanece durante gran parte del año con aguas tranquilas en las que las naves se encuentran a salvo.

En definitiva, va a ser la estructuración realizada por los movimientos alpinos, en el Mioceno superior, de los relieves bélicos orientales, la que marque las directrices que condicionarán la circulación viaria terrestre y portuaria de este territorio, en la actualidad convertidos en uno de los impulsores del desarrollo socioeconómico de la ciudad.

RÉSUMÉ

L'emplacement géographique de la ville d'Alicante dans le sud-est péninsulaire est motivé par la convergence sur place de deux voies naturelles de pénétration vers l'intérieur et par son contact privilegié avec la Méditerranée. En éffet, la ville se trouve protégée par les réliefs bétiques des incidences telles que des vents du Lévant, de sorte que la baie auprès de laquelle se trouve la ville offre des eaux calmes pendant une très grande partie de l'année, où les embarcations se trouvent à l'abri.

Bref, c'est la structure des reliefs bétiques orientaux, résultante des déformations alpines pendant le Miocene Supérieur qui conditionne la circulation routière et maritime sur le térritoire, convertie de nos jours un stimulant du développement socio-économique de la ville.

\section{Introducción}

Analizar lo que fue en el pasado y será en el futuro la causa y razón de ser de la ciudad de Alicante, encrucijada de caminos, debe considerarse estudio prioritario a fin de realizar una planificación adecuada a sus exigencias.

\footnotetext{
${ }^{1}$ Conferencia pronunciada el día 28-XI-1989, dentro del ciclo «Alicante, una ciudad hacia el futuro».
} 
La posición geográfica que ocupa la ciudad en el litoral levantino peninsular viene justificado por la convergencia sobre este lugar de dos vías naturales de penetración hacia el interior y su contacto con el Mediterráneo, al abrigo de los relieves béticos que la libran de las incidencias puntuales de los vientos de levante, de manera que la bahía junto a la que se asienta permanece durante gran parte del año con aguas tranquilas en las que las naves se encuentran a salvo. En definitiva, va a ser la estructuración realizada por los movimientos alpinos, en el Mioceno Superior, de los relieves béticos orientales, la que marque las directrices que condicionarán la circulación viaria -terrestre y portuaria- de este territorio.

La orografía quedó organizada con un eje longitudinal deprimido que desde los Arenales del Sol penetra cerca de $300 \mathrm{~km}$ hacia el SW -Fosa Intrabética- convirtiéndose en una arteria viaria de gran importancia desde la prehistoria hasta la actualidad. Al mismo tiempo, la fracturación transversal a la dirección del plegamiento bético que se produjo desde esta fosa hasta la Meseta en su extremo más oriental -Falla del Vinalopó- permitió la penetración desde el litoral hacia La Mancha y los valles longitudinales que ella corta, de manera que es otra vía natural de acceso al interior en dirección N., convergente sobre la primera en el Altet. La proyección hacia el E. y S. tropezaba de un lado con las terminaciones béticas -Sierras de Aitana y Bernia- y de otro con el mar Mediterráneo, de manera que sólo se podía continuar por vía marítima, por lo que se hizo necesaria la fundación de un puerto en el que se produjera la ruptura de carga para seguir en uno u otro sentido. Es así como nace la ciudad de Alicante, en asentamientos comprendidos entre la Albufereta Benalúa, para finalmente, por razones defensivas en época musulmana asentarse definitivamente al pie del Benacantil, al borde de las confluencias de las tres vías de comunicación, por lo que no cabe duda de que estamos ante uno de los ejemplos más claros de fundación por encrucijada de caminos.

De cara al futuro de esa encrucijada que es la ciudad de Alicante y del territorio inmediato organizado en función de ella, se impone la necesidad de realizar estudios que tiendan a mejorar las infraestructuras de relación de las redes viarias convergentes sobre esta área metropolitana. Cualquier planificación territorial ha de sustentarse en la confluencia de las distintas redes de transporte y los medios encargados de hacer la articulación necesaria de las variadas conexiones que estas mallas deben de presentar en el entramado urbanístico del núcleo alicantino, a fin de alcanzar la máxima fluidez de circulación con un número creciente de vehículos y de unidades de carga de modo que los diferentes medios de transporte muevan el mayor contingente posible de mercancías y viajeros sin llegar a producir el colapsamiento de algunas de las vías, como sucede en la actualidad.

Por ello, siendo conscientes de que el modo de producción de libre cambio ha consolidado un modelo territorial específico, que en nuestro caso presenta una distribución espacial de núcleos urbanos con cimentación histórica a la que se adosan barios residenciales y polígonos industriales, su resultado ha sido el de la configuración de áreas metropolitanas y urbanas más o menos definidas, conectadas entre sí por una red vial de comunicación poco desarrollada y deficientemente adaptada a las demandas que las estrategias del capital hoy exigen. Estamos, pues, en un momento decisivo para la ciudad de Alicante, que si bien por su posición geográfica y el desarrollo en el siglo XVIII de una red de caminos que desde ella van a partir la convierten en un lugar de convergencia de gran valor geoeconómico, se tendrá que adaptar a las peticiones del capital (público y privado) y de la sociedad para no perder la oportunidad histórica que la proyecte al siglo XXI.

A tal fin hay que ofrecer un territorio inmediato bien intercomunicado, local, regional y estatalmente, con superficies adecuadas para las instalaciones industriales y de servicios, junto a barrios con buena disposición a acoger la mano de obra que aquellas demandan, completado en ese espacio con superficies dedicadas a la práctica del ocio y promoción 


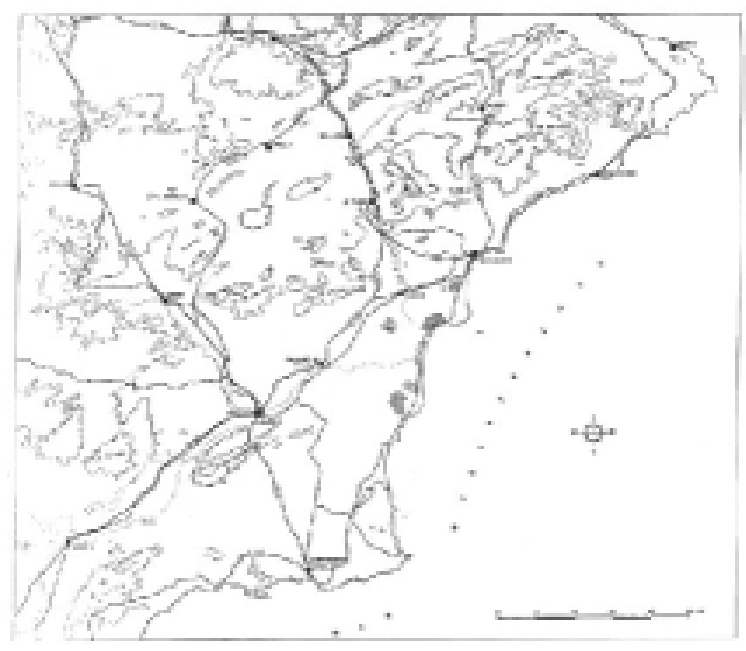

GRÁFICO 1. Mapa de situación de Alicante. En él se puede apreciar el condicionamiento de las unidades geomorfológicas béticas respecto a las principales vías de comunicación que convergen sobre esta ciudad. 
cultural. Negar estas planificaciones no querer reconocer la evidencia de que la jerarquización del crecimiento de las áreas metropolitanas depende del papel que juega cada país y cada región en el conjunto mundial, ya que el aparato económico local está cada vez más sometido a las estrategias de producción que decide el capital. En el caso alicantino es evidente que un desarrollo socioeconómico está supeditado a él, pues sus actividades agrícolas, industriales y turísticas son accionadas en muchos casos por empresas privadas cuyas decisiones son tomadas a miles de kilómetros, donde están los mercados consumidores, mientras que las de infraestructuras viarias son tomadas por los gobiernos regionales y estatales, por lo que se entra en una dinámica peligrosa si los responsables locales no saben actuar con rapidez y agilidad para exigir a instancias superiores dotación al territorio de la infraestructura necesaria para que quede bien organizado e intercomunicado, tanto a nivel interno, regional y estatal como mundial.

$\mathrm{Si}$ bien los condicionantes son propicios en el caso alicantino, tal como se desprende de la posición geográfica y evolución histórica de la ciudad, así como de la serie de obras programadas y efectuadas en lo que va de siglo, todavía son muchos los aspectos a los que se debe prestar una pronta atención para no perder esta oportunidad de planificación previsora del desarrollo futuro. A mi modo de ver, y a fin de entrar a su estudio, los temas prioritarios a resolver son los siguientes:

1. ${ }^{\circ}$ Completar la red de autopistas-autovías que convergen sobre la ciudad: Autovías de Torrevieja, la de Alcoy-Hoya de Castalla y autopista de Madrid.

2. ${ }^{\circ}$ Mejora de la red ferroviaria que une a La Encina y Murcia, en ambos casos con desdoblamiento de vía, y en el segundo además con electrificación, sin olvidar la posibilidad de unir estos itinerarios a la red de alta velocidad prevista para todo el territorio español.

3. ${ }^{\circ}$ Definición de la vocación portuaria de Alicante y, en el caso de optar por la realización de un puerto de mercancías, interrelacionar los planes de Ordenación Urbana con los previstos para el puerto, a fin de no crear problemas de circulación y servidumbre en el interior de la ciudad.

4. ${ }^{\circ}$ Convertir el aeropuerto de Alicante en suprarregional, mediante su conexión con vías rápidas de transporte terrestre, de manera que quede integrada su utilización como medio rápido para hacer largos desplazamientos.

\section{La red de autopistas-autovías}

La entrada en funcionamiento de las autovías de Alicante a Murcia y Almansa supondrá a corto plazo, la integración plena del territorio provincial en las grandes redes de comunicación nacionales y, sobre todo, europeas, ya que prolongará hacia el sur el gran eje de comunicaciones que es la autopista A7, vital para las exportaciones de productos agrarios e industriales y, sobre todo, para la llegada de los flujos turísticos que tanta repercusión tienen en el desarrollo socioeconómico costero.

Ahora bien, a pesar de estos logros todavía quedan pendientes, a nuestro entender, dos grandes obras de infraestructura viaria que son la construcción de una carretera que, con algún tramo de túnel, contribuya a lograr un buen acceso a las comarcas interiores -Hoya de Castalla y Valles de Alcoy- y, de otro lado, la construcción de un tramo de autovía que partiendo del límite con la provincia de Murcia enlace las tierras litorales del sur, e incluso canalice hacia Alicante gran parte de la actividad agraria y turística del litoral murciano.

Respecto de la autovía de Torrevieja, disiento del planteamiento que se hace del trazado como desdoblamiento de la N-332, ya que esto sólo seria posible en algunos tramos de ella, puesto que en otros, por afectar a zonas con un alto grado de urbanización 


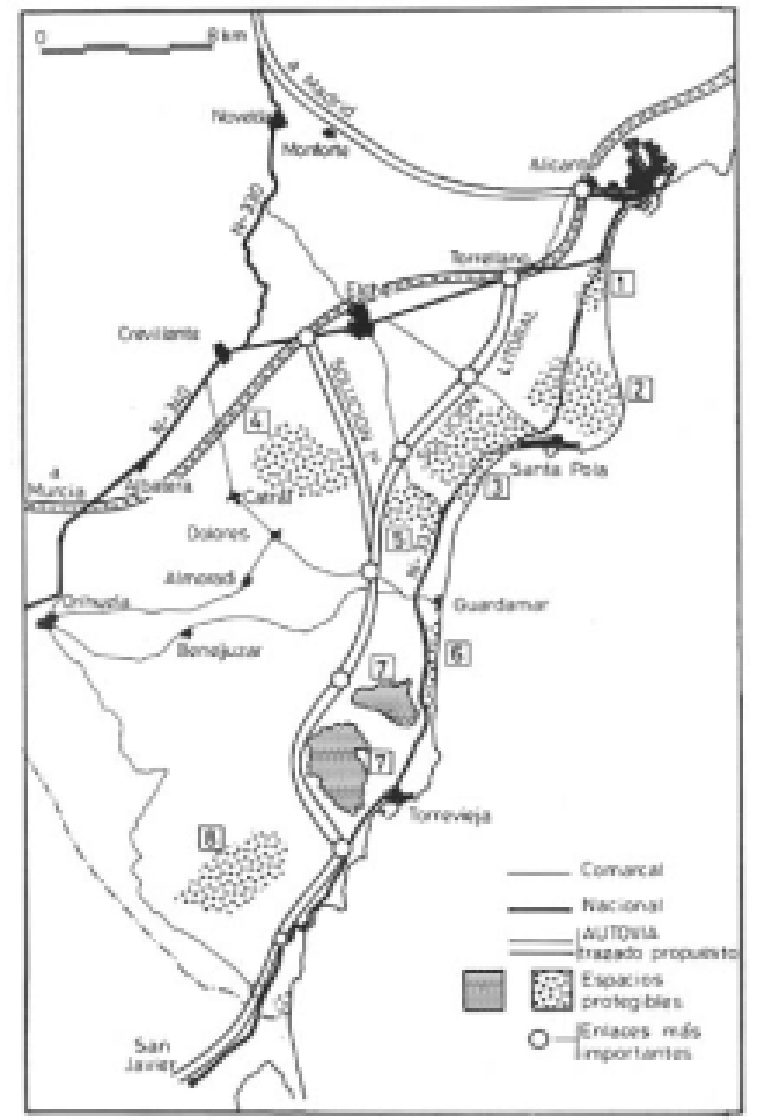

GRÁFICO 2. Propuesta de trazados autovía de Torrevieja, con indicación de los espacios naturales protegibles: 1. Saladar de Agua Amarga; 2. Cabo de Santa Pola y humedales próximos; 3. Albufera de Elche; 4. Laguna Hondo de Crevillente; 5. Sierra del Molar; 6. Dunas de Guardamar-La Mata; 7. Lagunas de La Mata y Torrevieja; 8. Pinar del Río Nacimiento. 
y congestión turística, o discurrir por áreas de gran valor medioambiental, no sería deseable contribuir a aumentar más el impacto pernicioso que este tipo de obras introduce en los ecosistemas frágiles.

Además, en busca de una mayor funcionalidad, e incluso de ahorro económico, se propone un nuevo trazado. Este se iniciaría en el limite sur-provincial, en el término municipal de El Pilar de la Horadada, enlazando con la proyectada autovía del litoral murciano y circunvalación de San Pedro del Pinatar, para continuar, como desdoblamiento de la N-332 hasta la urbanización del Plan Parcial Punta Prima; de ahí, mediante un nuevo trazado, bordearía por el oeste las lagunas de Torrevieja y La Mata, discurriendo por la Marquesa, Los Montesinos y Campo de Guardamar, para cruzar el Segura al E. del municipio de Rojales y San Fulgencio y desde aquí dirigirse hacia los Carrizales, sin afectar al espacio lagunar inmediato. En este punto se puede optar por dos soluciones:

a) La más corta se dirigiría hacia el norte por el Derramador, La Matola y La Algueda, en busca del nudo y salida W. de Elche, construido sobre la autovía de Alicante a Murcia.

b) La solución litoral implica la continuación del trazado paralelo a la costa, discurriendo por las partidas de Asprillas, Las Bayas y Valverde hasta enlazar con el nudo de salidas de la autovía Alicante-Murcia al aeropuerto y enlace con la circunvalación de Alicante en Torrellano. La justificación de un trazado alejado unos $10 \mathrm{Km}$ de la línea de costa entre el límite sur del municipio de Torrevieja y el nudo de Torrellano se entiende por varios motivos:

1. Se trata de una vía de primer orden encargada de canalizar y redistribuir flujos de tráfico que tienen su origen y destino en lugares bastante alejados entre sí.

2. Con ello se configuraría un esquema de acceso perpendicular a la costa, de acuerdo con los criterios de una adecuada ordenación y vertebración de los espacios costeros.

3. La vinculación entre los núcleos urbanos tradicionales y los nuevos asentamientos turísticos quedaría liberada de los flujos de paso, con lo que las relaciones funcionales se verían perfectamente atendidas por la actual N-332, que hoy en día actúa como eje estructurante de la dorsal urbano-turística entre Arenales y El Mojón.

4. El trazado interior no obligaría a realizar obras que dificulten la expansión del continuo urbano de los núcleos costeros, que de otra forma verían constreñidas sus posibilidades de crecimiento. A mayor abundamiento, la falta de previsión de una red de accesos de este tipo en municipios de la talla de Torrevieja, dificultaría extraordinariamente la posibilidad de trazar una autovía a pocos cientos de metros de la costa, por lo que lo más deseable es liberar del tráfico de paso la actual CN-332.

5. El trazado propuesto se aleja de las zonas que es preciso proteger en atención a su alto valor ecológico y medio ambiental, tales como el Saladar de Agua Amarga, el Cabo de Santa Pola, la Albufera de Elche y sus aledaños húmedos, el Hondo de Dolores-Crevillente, las lagunas de Torrevieja y la Mata y las líneas de monte bajo y pinares de Las Majadas, Dehesa de Campoamor y curso medio y alto de los ríos Seco y Nacimiento.

6. El cruce del valle del Segura por el E. de los municipios de Rojales y San Fulgencio permitiría la realización de un nudo de enlace que vertebraría el tráfico de la Vega Baja y litoral de Guardamar.

El interés de estas obras desde el punto de vista económico se fundamenta en los siguientes apartados:

a) Potenciación del área metropolitana de Alicante como nudo de comunicación, una vez concluyan las obras de las citadas autovías. Así, contribuirá de manera decisiva al reforzamiento de la ciudad como centro de servicios, no sólo provincial sino extrarregional, al convertirse el aeropuerto, el puerto y el nudo ferroviario (merced a la electrificación de la vía entre La Encina y Alicante), en centro de comunicación aérea, terrestre y 


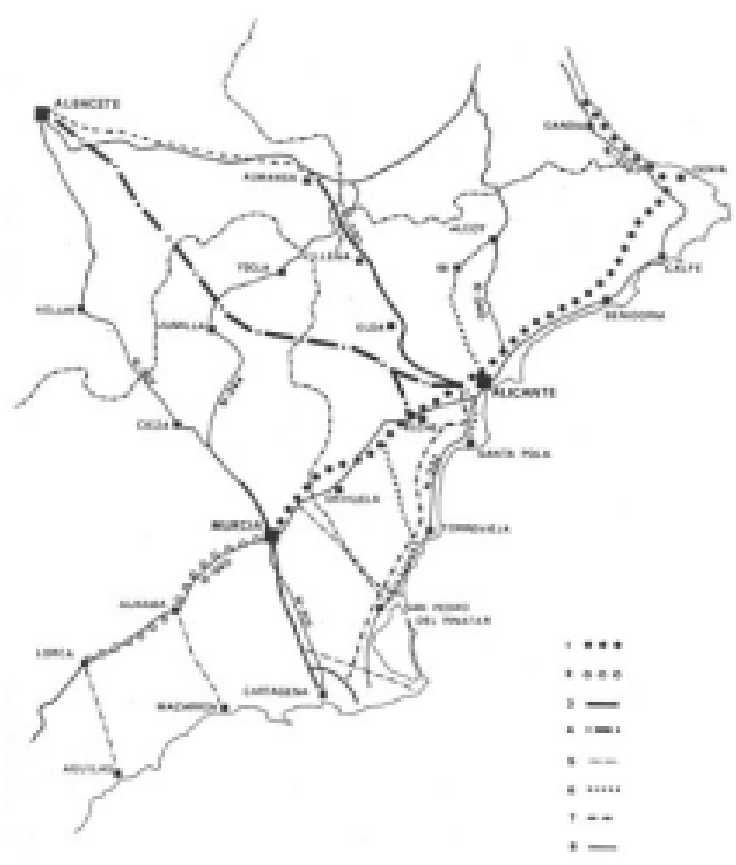

GRÁFICO 3. Red viaria básica actual y sus posibles mejoras futuras: 1. Autopista A-7 (E.15); 2. Prolongación hacia Andalucía de la A-7 en construcción; 3. Autovías a Alicante-Madrid y Murcia Cartagena; 4. Posible trazado de la futura autopista Alicante-Madrid; 5. Autovías en construcción y en proyecto desde la A-7 a la costa en la Comunidad de Murcia; 6. Propuesta de posibles trazados de las autovías alicantinas desde la A-7 a la costa y valles de Alcoy; 7. Trazado previsto por el MOPU para la autopista Alicante-Cartagena; 8. Carreteras nacionales. 
marítima que serviría también a la Comunidad Murciana y parte de Castilla-La Mancha, que hasta ahora, por la carencia de vías rápidas que articulen los polos de transporte, no han tenido el desarrollo que sería deseable.

b) La autovía del Sur jerarquizará y organizará la estructura territorial del amplio espacio que se extiende desde el litoral de Elche, Santa Pola, Guardamar del Segura, Torrevieja, litoral de Orihuela y El Pilar de la Horadada, espacio que registra en la actualidad los mayores índices de crecimiento y muchas carencias de infraestructura. Pero, más aún, la nueva vía conectará con la autovía costera prevista en la Comunidad Murciana entre Cartagena y San Pedro del Pinatar, desempeñando la misma función para el espacio turístico de La Manga y el Mar Menor. Todo ello sin olvidar que su discurrir afectará a las áreas de transformación agrícola más avanzada de Alicante y Murcia, con unas necesidades de transporte rápido por carretera hacia los mercados europeos.

La propuesta de construcción de una autovía que una Alicante con La Hoya de Castalla y Valles de Alcoy, está justificada por el viejo anhelo de acortar en el tiempo el viaje hacia esas tierras. La obra está prevista en el Avance de Obras Públicas de la comunidad Valenciana de 1986, como la terminación meridional del eje central que con dirección $\mathrm{N}-\mathrm{S}$, se ha proyectado para vertebrar viariamente la Comunidad.

Sin embargo disentimos del planteamiento realizado por algunas instituciones de constituirlo con un túnel que atraviese la Carrasqueta, por varias razones:

a) Al hacerlo por la Hoya de Castalla se beneficia a otra comarca industrial alicantina al tiempo que su conexión con la autovía de circunvalación de Alicante se podría materializar en las proximidades de San Vicente, descongestionando el área de San Juan.

b) El costo material sería menor al evitar la construcción del túnel.

Muchas de las matizaciones referidas a la autovía de Torrevieja serán válidas para esta última. De esta manera el Sur de la Comunidad Valenciana presentaría una red de carreteras bien vertebradas, válidas para hacer la interrelación con tierras próximas de Murcia y La Mancha.

En 1990 se concluyó el tramo de autovía de Levante (Madrid-Alicante) desde Almansa a Alicante, con una longitud de $75 \mathrm{Kms}$. Las obras consistieron, en realidad, en el desdoblamiento de la calzada de la vieja carretera nacional 330, separadas por una mediana dura de unos dos metros de anchura, muy alejada de las exigencias de la CEE (de 5 metros de mediana en seto vivo, arcenes exteriores de 2,5 metros de ancho, radio mínimo en curva horizontal de 450 metros y pendientes del 5\%), por lo que las velocidades máximas permitidas de $100 \mathrm{Km} / \mathrm{h}$, son similares a las de su predecesora de tipo Redia proyectada en 1967. El resultado ha sido una inadecuación de dicha autovía a las necesidades reales del tráfico en este tramo, con un saldo trágico, en su primer año de funcionamiento, de 28 muertos y 78 heridos graves en 115 accidentes (ABC de 29-04-90). Las previsiones, por otro lado, apuntan a una pronta saturación de vehículos circulantes en las horas laborables entre Villena y Alicante.

En definitiva, con esta obra de un costo para $75 \mathrm{Kms}$ de 9.000 millones de pesetas, no se tuvo en cuenta las previsiones de construcción de una autopista de peaje Madrid-Alicante, la cual tendría que construirse de cara al año 2000; sobre todo en el trayecto Albacete-Alicante como alternativa a la autovía, si no olvidamos que la salida natural de la capital del estado es el litoral alicantino, y mediante una vía rápida, tipo autopista, esa distancia a una media de $130 \mathrm{Km} / \mathrm{h}$ se haría en sólo tres horas. Así, los espacios de ocio litorales tienen unos potenciales usuarios en los habitantes de la gran aglomeración madrileña, como ya lo han sido tradicionalmente desde finales del siglo XIX.

Se impone, pues, volver a insistir en la construcción de la Autopista Madrid-Alicante. Ahora bien, su planteamiento hay que hacerlo con una ideología economicista suprarregional 
a fin de interesar en su construcción no sólo aquellas autonomías situadas en sus extremos, como normalmente se hace, pues de esta forma se olvida la rentabilidad de una obra y entonces se choca con la oposición de unos y el desinterés de los que harían la construcción y su posterior explotación. Por estas razones el trazado de esta autopista tendría interés, también, para las Comunidades de Castilla-La Mancha y Murcia, siempre que ésta siguiese en parte el viejo camino real, funcional hasta mediados del siglo XIX que acortaba el trayecto entre Albacete y Alicante, sustancialmente al descender desde Chinchilla a Montealegre-Yecla-Pinoso-Monóvar.

La propuesta sería construir una autopista con el siguiente itinerario: Albacete, Chinchilla, Pétrola, Fuente-Álamo, Yecla, Venta Quebrada, Monóvar, Elda. Varias son las ventajas a señalar en defensa de este trazado: 1) Se acorta la distancia entre Albacete y Alicante en $30 \mathrm{kms}$; 2) Las pendientes a salvar son menores a las de la actual carretera nacional, pues en ningún caso se superarían los 750 metros de altitud; 3) Al SE de Jumilla se podría entroncar a esta autopista con la red de la Comunidad Murciana que también la utilizaría en sus relaciones con Madrid; 4) Al realizar la penetración en el Valle del Vinalopó por Pinoso y Monóvar evitaría los problemas de acercarse a las aglomeraciones poblacionales de Elda-Petrel-Monovar, de la que quedaría alejada unos 6 ó 7 kms; 5) Su finalización sobre la A-7 se haría hacia el SW de Elche en la convergencia sobre ella de la posible autovía del S., por lo que desde allí se procedería a la captación de los tráficos de origen en el Bajo Vinalopó, Bajo Segura y parte del litoral turístico murciano; 6) Para acceder a la ciudad de Alicante y litoral Norte provincial se podría hacer un ramal hacia el Este en digitalización que conectara con la autovía de penetración en esa dirección y que incluso duplicaría en un recorrido de 10 a $12 \mathrm{kms}$.

De esta forma quedaría estructurada una sólida red vial de penetración hacia el litoral alicantino con la A-7 y la autopista de Madrid complementada con las redes de los niveles nacional y regional, sin olvidar las planificaciones urbanas que tienen la necesidad de contemplar variados enlaces con estos tres estadios viales, para evitar los problemas circulatorios del interior de ellos.

\section{Nueva vocación portuaria}

El puerto de Alicante está sufriendo en la actualidad una disminución en su tráfico de mercancías, se ha querido buscar la causa de ello en la falta de interés que se presta a la modernización de sus instalaciones y en unas actuaciones discriminatorias desde la Administración Autonómica en favor del puerto de Valencia. Si han existido éstas, realmente no han tenido la responsabilidad que se le atribuyen, puesto que la atonía experimentada en el movimiento portuario alicantino hay que justificarla, a mi modo de interpretar la cuestión, en los avances tecnológicos habidos en los barcos y en las formas de organizar los circuitos de transporte por las compañías consignatarias de buques.

En el caso alicantino han sido las nuevas tecnologías de los barcos y las malas condiciones de la bahía las que impiden la adecuación a las nuevas exigencias del transporte marítimo, quedando limitadas sus posibilidades a recibir barcos de tonelajes inferiores a las $15.000 \mathrm{Tm}$ y de calados y esloras de tipo medio a pequeño, resultando imposible el atraque de los grandes portacontenedores y graneleros que superen ese arqueo.

En esa tesitura convendría plantearse el futuro del puerto en relación a una vocación de puente de enlace con el Norte de África y Archipiélago Balear, para tener la presencia en sus aguas de buques rápidos -ferrys-, que trasporten viajeros y en sus bodegas puedan acceder los camiones TIR, autobuses y automóviles. Aquí se está en condiciones de

completar y centralizar tráficos que requieran una movilización rápida sin producir en la práctica ruptura de carga. 


\section{Mejoramiento de la red de ferrocarriles}

El ferrocarril, que ya jugó en el siglo XIX un papel importante en el desarrollo socioeconómico de la ciudad de Alicante y en parte de su provincia, está de nuevo llamado a dar otro gran impulso al papel que va a hacer esta ciudad en el conjunto de las comunicaciones del litoral levantino peninsular. Así, a este respecto, afirmo Karel Van Miert, comisario de la C.E.E. el día 22-XI-1989 en Bruselas, al dar cuenta del programa Rail 92 la necesidad de relanzar el transporte ferroviario como un medio de futuro, aunque ha perdido parte de mercado, puesto que se considera la única alternativa europea para paliar «un tráfico por carretera saturado y la congestión aérea».

En este orden de cosas, para el caso alicantino, es vital la actuación inmediata sobre los trazados La Encina-Alicante y Murcia-Alicante, acometiendo la instalación de una segunda vía, construida sobre traviesas de doble uso para que llegado el caso se pueda hacer la rápida adaptación al ancho europeo. Esta demanda viene dada por el hecho de que disponiendo en la actualidad de material móvil que puede alcanzar los $160 \mathrm{Km} / \mathrm{h}$ en estos trayectos, apenas si se superan los 90, al tiempo que los cruces de diferentes unidades de direcciones contrarias actúan como cuellos de botella.

Si esto se consiguiera se podría dar una batalla a favor del ferrocarril en las distancias cortas y medias, pues los trayectos Madrid-Alicante se podrían reducir a unas 2,40 horas; ValenciaAlicante a 1,20 horas y Murcia-Alicante a sólo 35 ó 40 minutos. De manera que no habría medio de transporte terrestre y aéreo que pudiese competir con él.

Además el transporte ferroviario debe de integrarse con el aéreo, si se acomete estas transformaciones que se proponen y para ello sería necesario realizar una pequeña modificación de no más de 3 kilómetros en el trazado de las vías de Alicante-Murcia para que se acercase hasta la terminal de viajeros del aeropuerto y de esta forma poder ofrecer un servicio conjunto, tal como existe en algunos aeropuertos europeos.

La llegada del tren de alta velocidad puede ser una realidad a más largo plazo cuando se articule la vía del litoral mediterráneo español que se pretende hacer descender hasta Murcia. Pero de momento ya sería un gran logro duplicar las velocidades actuales y alcanzar ese óptimo de $\operatorname{los} 160 \mathrm{Km} / \mathrm{h}$ que dice RENFE poder realizar en la actualidad.

\section{Hacia un aeropuerto suprarregional}

El Altet, con sus $300 \mathrm{Ha}$. de terrenos ocupados, de los cuales unos $40.000 \mathrm{~m}^{2}$ corresponden a las dependencias de sus terminales de viajeros y mercancías, entraría dentro de una categoría de aeropuerto a nivel internacional de tipo medio. Por lo que está considerado muy bien proporcionado en relación con su movimiento, sin apenas deficiencias técnicas, salvo algunas de ayuda a la navegación en aproximación a sus pistas, que se suplen normalmente de visu por las buenas condiciones meteorológicas que se dan casi a diario en esta región.

La ubicación del aeropuerto se hizo siguiendo los criterios al uso en este tipo de instalaciones, es decir, proximidad a grandes urbes, pero al mismo tiempo buscando zonas que no presenten concentraciones de población en sus inmediaciones. Por esta razón, cuando se proyectan nuevos aeropuertos para grandes ciudades se tiene que ir a ubicarlos 


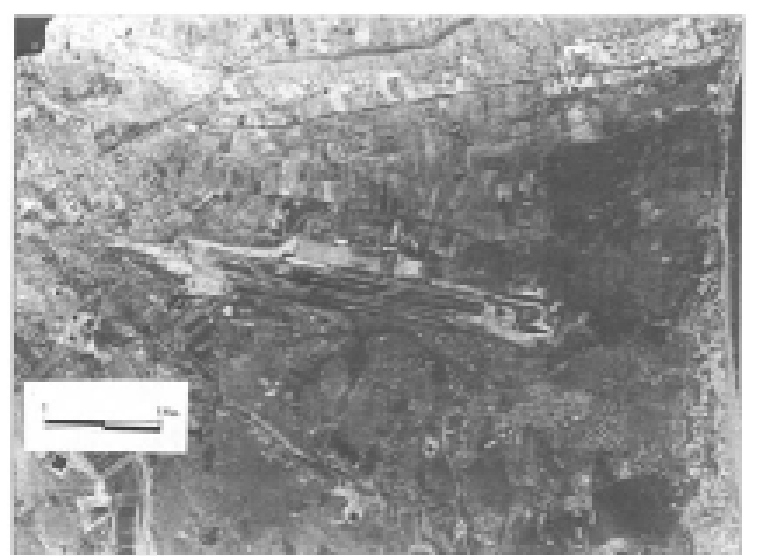

GRÁFICO 4. Vista del aeropuerto y de las vías de acceso al mismo. 
a áreas distantes de ellas entre 50 y $80 \mathrm{Km}$, mientras que en el caso de el Altet se pudo emplazar a sólo $8 \mathrm{Km}$ de Alicante y a unos 12 de Elche, con una zona de influencia en semicírculo de 200 $\mathrm{Km}$ de radio, en la que quedan incluidos por NE. todos los grandes centros turísticos alicantinos, por el S. se llega hasta el complejo de La Manga-Mar Menor de Murcia, al W. se engloba a la capital de aquella región y por el N. comprende el industrioso Valle del Vinalopó. Se trata de un área de influencia de alta densidad demográfica -casi dos millones de habitantes- y con una economía floreciente alrededor de las actividades agrícolas, industriales y mercantiles que la convierten en una de las más dinámicas de España. Razones éstas más que suficientes para justificar su buen emplazamiento, que todavía se revaloriza mucho más e incluso acredita su presencia en la zona, si se examina la procedencia de los pasajeros -en un $75 \%$ turistas- que tenían como destino preferente Benidorm y sus aledaños.

Estos últimos datos indican que la importancia del aeropuerto alicantino deriva de su situación central respecto a la zona costera próxima, ya que algo menos de una cuarta parte de su movimiento se justifica en relación con las otras actividades socioeconómicas de su zona de atracción. Tiene que soportar una dura competencia del ferrocarril y de los autobuses, más rentables desde un punto de vista de infraestructuras que necesitan para su funcionamiento, a la vez que ya empiezan a competir desde la variable tiempo, que era la que en parte se esgrimía para justificar su utilización en estas distancias. Por tanto la solución está en acercar a esos potenciales viajeros rápidamente a las terminales aéreas y para ello es necesario disponer de una adecuada red de carreteras, que por desgracia es la deficiencia más notable que el aeropuerto alicantino ha presentado hasta la actualidad.

Los planificadores de este tipo de instalaciones y los estudiosos de estos temas han considerado que todo buen aeropuerto requiere accesos terrestres fáciles y rápidos, no sólo para el usuario del transporte, sino también para los trabajadores de él. Los primeros pueden agruparse en tres categorías: viajeros de negocios, turistas y expedidores de mercancías. Los que van de viaje comercial consideran fundamentalmente el tiempo como algo primordial, por lo que la rapidez de circulación entre las terminales aéreas y los centros de las ciudades es imprescindible. Los turistas son menos exigentes en cuanto al tiempo, salvo que vengan de fin de semana, que es una de las modalidades de turismo que se quiere fomentar durante el invierno en Benidorm. Los expedidores de mercancías perecederas demandan, asimismo, agilidad en la aproximación a las terminales de carga. Los segundos, los trabajadores del aeropuerto, necesitan fluidez en la circulación de los accesos, ya que constituyen un colectivo bastante importante -100 por cada millón de pasajeros-, de forma que en horas punta contribuyen con su desplazamiento al trabajo y el de sus familiares a aumentar el tráfico, ya que normalmente localizan sus viviendas en las cercanías de estas vías y del aeropuerto. Como se ve, el planteamiento teórico de la ordenación de la accesibilidad exige fluidez y rapidez, porque de otra forma cualquier instalación de este tipo está condenada a muerte por inanición.

Si los viajeros tienen dificultades para llegar a El Altet, los envíos de mercancías también las padecen, puesto que algunas, como las frutas y flores, proceden de la Vega Baja y Campo de Cartagena, y aquí la red de carreteras para aproximarse al aeropuerto todavía presenta mayores deficiencias que en los casos anteriores.

Con esta situación gravitando sobre el futuro del aeropuerto alicantino, en los últimos meses se ha observado un movimiento en algunos sectores de intereses económicos, sociales y políticos que apuntaban hacia una defensa chauvinista del aeródromo, e incluso se llegó a decir que se había realizado una descalificación en favor de su vecino de Manises. La realidad no es tan simple, sino que depende de las condiciones que éste tenga para poder atender a la demanda que sobre él recae. 
La realización de la infraestructura de carreteras en las inmediaciones del El Altet, sobre todo circunvalación de Alicante, viene a solucionar una problemática de viabilidad para el futuro, si es que verdaderamente se quiere hacer desparecer el fantasma que sobre él pesa y de esta forma, también, se contentarían los intereses murcianos que ya no demandarían la construcción de su propio aeropuerto. A pesar de ello, no hay que olvidar que el tráfico interior debe tender a disminuir porque ya empieza a dejarse sentir la regla de oro del transporte, de que en las distancias medias y cortas -en nuestro caso Madrid y Barcelona- el tren tiene la supremacía, como se ha demostrado ya en algunos trayectos de la red nacional que para Alicante se dejará sentir de forma notable, cuando se realice la doble vía de ferrocarril de los tramos que le une con La Encina y Murcia.

Esta modernización de accesos al aeropuerto implica también la mejora de los viales periurbanos de Alicante y Elche, porque en estos espacios se producen atascos circulatorios incididos por la presencia de él, como se indicó anteriormente y que dada su situación actual harían más penosas sus travesías y, de otro lado, se hace necesaria la construcción de una estación de ferrocarril en sus inmediaciones.

Habría que apuntar, finalmente, que todas estas obras requieren grandes inversiones del sector público, por lo que hay que concebirlas con visión de futuro y sobrepasando límites comarcales y regionales. Por ello El Altet debe concebirse como el aeropuerto del Sur de la Comunidad Valenciana y de la Región Murciana.

Todas estas propuestas deben de ser contempladas en una conjunción de intereses municipales plurales, fundamentalmente de Alicante y Elche, a fin de evitar un planeamiento irracional de sus superficies, del que más tarde no nos tengamos que arrepentir. Como ya he apuntado en varias ocasiones, no se trata de hacer una área metropolitana por conurbación de decisiones tomadas en las más de las veces, de manera contradictoria, que nos lleven a una situación en la que cualquier mejora suponga una operación quirúrgica que haga sangrar el mal entramado urbano que de otra forma se podría generar. Hay que tomar decisiones en la actualidad con visión de futuro, contemplando la conservación de espacios naturales y agrícolas de interés como es el caso de los palmerales, tan necesarios como pulmones verdes dentro o cerca del solar de las ciudades.

\section{Bibliografía}

ABELLÁN GARCÍA, A.: «Los ferrocarriles del Sureste. Estudio Geográfico». Instituto «Juan Sebastián Elcano». C.S.I.C. 1979.

CANO GARCÍA, G. M.: «El transporte aéreo en España». Ed. Ariel. Barcelona, 1980.

DACHARRY, M.: «Géographie du Transport aérien». Ed. Litec, París, 1981.

FERNÁNDEZ DURÁN, R.: «La quiebra de la política de transportes». I.C.E. Madrid, 1981. MORALES GIL, A.: "Las comunicaciones en Murcia ante el desarrollo económico regional». Papeles del Departamento de Geografía n..$^{\circ}$ 4. Univ. Murcia, 1974.

MORALES GIL, A.: "La red viaria en el área metropolitana de Alicante-Elche». Investigaciones Geográficas n. ${ }^{\circ} 1$. Universidad de Alicante, 1983.

MORALES GIL, A.: «Accesibilidad por carretera al aeropuerto de Alicante». Canelobre n. ${ }^{\circ}$ 5. Inst. . Juan Gil Albert. Alicante, 1985.

PREVASA: «Estudios básicos para la ordenación del territorio de la Comunidad Valenciana». Caja de Ahorros de Valencia, 1982.

SANCHIS DEUSA, C.: «El transporte en el País Valenciano». Ed. Alfonso el Magnánimo. Valencia, 1988.

VERA REBOLLO, J. F.; MORALES GIL, A. y otros: «Libro blanco del Turismo en la Costa Blanca». Cámara Oficial de Comercio, Industria y Navegación de Alicante, 1991. 